\title{
UNIVERSAL MATRICES AND STRONGLY UNBOUNDED FUNCTIONS
}

\author{
Piotr KoszMider
}

\begin{abstract}
Fix an uncountable cardinal $\lambda$. A symmetric matrix $M=\left(m_{\alpha \beta}\right)_{\alpha, \beta<\lambda}$ whose entries are countable ordinals is called strongly universal if for every positive integer $n$, for every $n \times n$ matrix $\left(b_{i j}\right)_{i, j<n}$ and for every uncountable set $A=\{a: a \in A\} \subseteq[\lambda]^{n}$ of disjoint $n$-tuples $a=\left\{a_{0}, \ldots, a_{n-1}\right\}<$ there are $a, a^{\prime} \in A$ such that $b_{i j}=m_{a_{i} a_{j}^{\prime}}$ for $0 \leq i, j<n$. We go beyond the recent dramatic discoveries for $\lambda=\omega_{1}, \omega_{2}$ and address the question of the possibility of the existence of a strongly universal matrix for $\lambda>\omega_{2}$. Due to the undecidibility of some weak versions of the Ramsey property for $\lambda \geq \omega_{2}$ the positive answer can be at most consistent, but we show that some natural methods of forcing cannot yield that answer for $\lambda>\omega_{2}$. We use our method of "forcing with side conditions in semimorasses" to construct generically $\lambda$ by $\lambda$ strongly universal matrices for any cardinal $\lambda$. The results are proved in more generality, related concepts are investigated, some questions are stated and some application are given.
\end{abstract}

\section{Introduction}

1.1. General background. If $\lambda$ is an uncountable cardinal, by a restriction to the Ramsey property we will mean any partition $\left(P_{i}\right)_{i \in \kappa}$ of $[\lambda]^{2}$ such that there is no $[X]^{2} \subseteq P_{i}$ of full size $\lambda$ for any $i \in \kappa$ or a similar partition. ${ }^{1}$ It has been noted by Sierpiński in his paper [Si] of 1933 that there do exist restrictions to the Ramsey property even for $\lambda$ equal to the first uncountable cardinal and $\kappa=2$.

From this time on, it has been more and more clear that restrictions to the Ramsey property on some uncountable cardinal can be quite a useful tool in constructions of various mathematical structures. Recently new stronger results were obtained. They originated in a spectacular new partition of $\left[\omega_{1}\right]^{2}$ constructed by Todorcevic in his paper [T2] of 1987. In this partition, for every uncountable $X \subseteq \omega_{1}$, the set $[X]^{2}$ meets all parts $P_{i}$ for $i<\omega_{1}$. This was

Received April 16, 2001.

Some of the research leading to this paper, whose results were announced in 1996, was supported by NSF of USA Grant DMS-9505098 held by the author at Auburn University, AL, U.S.A., in the years 1995-1998.

Some of the research leading to this paper was supported by Fapesp fellowship No. 1997/10491-7 received at Universidade de São Paulo, SP, Brasil.

${ }^{1} \kappa$ could be any cardinal such that $\kappa<\lambda$ and the results of this paper will be stated usually in such a generality, however in this section we consider only the case of $\kappa \leq \omega_{1}$. 
followed by various readings of his proof (see [V3], [S2], [BSp]) which often included stregthenings needed for particular applications which later turned out to hold for the original partition (see [Be] and [T5]). These applications vary from p-groups ${ }^{2}$ or Banach spaces ${ }^{3}$ to constructions in quadratic vector spaces ${ }^{4}$

Aiming at strong restrictions to the Ramsey property above $\omega_{1}$ carries new dangers. There are two weak versions of the Ramsey property which may consistently hold for $\omega_{2}$, they are the Continuum Hypothesis (denoted $\mathrm{CH}$ in the sequel) in the disguise of the Erdös-Rado theorem ${ }^{5}$ and the Chang's Conjecture ${ }^{6}$ (denoted $\mathrm{CC}$ in the sequel).

Thus the situation for $\omega_{2}$ is more complex. In many consistency results restrictions to the Ramsey property on $\omega_{2}$ obtained assuming some (strong) negations of $\mathrm{CC}$ allow to construct forcing notions which add reals (hence the negation of $\mathrm{CH})$ and provide the generic constructions of the required structures.

Such are, for example, the constructions of a peculiar compact scattered space of [BS], a coloring of $\omega_{2} \times \omega_{2}$ nonconstant on the product of any two infinite sets of [T3], a set mapping on $\omega_{2}$ with no infinite free set of $[\mathrm{Kj}]$, a generalization of Shelah-Steprans Banach space result from [Ksz4], an increasing chain $\left(a_{\xi}\right.$ : $\left.\xi<\omega_{2}\right)$ in $\wp\left(\omega_{1}\right) /$ Fin such that $a_{\xi}-a_{\eta}$ is uncountable for $\eta<\xi$ of [Ksz2], a separation of any two disjoint closed sets in some topological spaces which gives the normality of these spaces of [EGKTT] and the Kurepa tree of [Ve].

On the other hand, Todorcevic (see [T4]) keeps developing canonical restrictions to the Ramsey property for $\omega_{2}$ which are weaker than the above-mentioned but exist without any special set-theoretic assumptions. This includes such results as the existence of a partition of triples $\left(P_{i}\right)_{i \in N}$ such that the triples of any uncountable subset of $\omega_{2}$ hit all the colours. However, these results of Todorcevic haven't found yet their applications outside combinatorics.

Further lies the land of cardinals beyond $\omega_{2}$. If we want to talk about restrictions to the Ramsey property relevant to the constructions at the level $\omega_{1}$ or $\omega_{2}$, not just shifting all the involved cardinals some levels up, we note that most known negations of CC needed for the generic constructions of sizes $\omega_{2}$ mentioned in the paragraph above such as a function with the $\Delta$-property, $\left(\omega_{1}, 1\right)$-morass, $\rho$-function do not generalize naturally above $\omega_{2}{ }^{7}$. The main result of this paper is the consistency of the possibility of generalizing to any cardinal $\lambda$ another

\footnotetext{
${ }^{2}$ (Shelah, Steprans [SS2]) There is an uncountable extraspecial p-group (for any prime $p$ ) with no uncountable abelian subgroup.

${ }^{3}$ (Shelah, Steprans [SS1]) There is a nonseparable Banach space where every operator is a scalar multiple of the identity plus a separable range operator.

${ }^{4}$ (Baumgartner, Spinas [BSp]) There is an uncountably dimensional full-angled quadratic vector space $(V, \Psi)$, in particular, it has no uncountable biorthogonal system of vectors.

${ }^{5}$ The Erdös-Rado theorem applies to $\lambda>2^{\omega}$ and implies that given partition $\left(P_{i}\right)_{i<\omega}$ of $[\lambda]^{2}$ there is an uncountable $X \subseteq \lambda$ such that $[X]^{2} \subseteq P_{i}$ for some $i<\omega$.

${ }^{6}$ Chang's Conjecture (see e.g. [Ka]) in particular implies that for every partition $\left(P_{i}\right)_{i<\omega_{1}}$ of $\left[\omega_{2}\right]^{2}$ there is an uncountable set $X \subseteq \omega_{2}$ such that $[X]^{2} \subseteq \cup_{i<\delta} P_{i}$ for some countable ordinal $\delta$.

${ }^{7}$ There exist also some highly profound principles called higher-gap morasses, however they are consistent with GCH and due to their level of complexity they are rarely used (see [D]).
} 
statement equivalent to the negation of CC for $\lambda=\omega_{2}$. This statement affirms the existence of certain functions $f:[\lambda]^{2} \rightarrow \omega_{1}$. We will also see that a new forcing method is needed to obtain these results as well as new combinatorial phenomena occur at this higher level. A few new simple applications are also given.

1.2. Notation. We follow the texts $[\mathrm{K}]$ and $[\mathrm{B}]$ and established notational customs, for example, if $A$ and $B$ are sets of ordinals, then $A<B$ means that $\alpha<\beta$ for all $\alpha \in A$ and $\beta \in B ; f \mid X$ denotes the restriction of a function $f$ to a set $X$ and $f^{\prime \prime} X$ denotes the image of $X$ under $f ; \operatorname{ordtp}(A)$ denotes the order type of the set of ordinals $A$. For an ordinal $\nu$ the notation $\left\{a_{i}: i<\nu\right\}_{<}$means that $a_{i}$ are ordinals and $a_{i}<a_{j}$ for $i<j<\nu$.

\subsection{Definitions and statements of the results.}

Definition 1. Fix an uncountable cardinal $\lambda$ and a regular cardinal $\kappa<\lambda$. A symmetric matrix $M=\left(m_{i j}\right)_{i, j<\lambda}$ whose entries are the ordinals less than $\kappa^{+}$ is called $a \lambda$ by $\lambda$

1) $\kappa^{+}$-strongly universal,

2) diagonally $\kappa^{+}$-strongly universal,

3) $\kappa^{+}$-strongly unbounded,

4) diagonally $\kappa^{+}$-strongly unbounded,

if and only if for every $\nu<\kappa$ and for every $\nu \times \nu$ matrix $\left(b_{i j}\right)_{i, j<\nu}$ with entries from $\kappa^{+}$, for every $\delta \in \kappa^{+}$and for every set $A=\{a: a \in A\} \subseteq[\lambda]^{\nu}$ of cardinality $\kappa^{+}$of disjoint $\nu$-tuples $a=\left\{a_{i}: i<\nu\right\}_{<}$there are distinct $a, a^{\prime} \in A$ such that for all $i, j<\nu$ the following hold respectively:

1) $b_{i j}=m_{a_{i} a^{\prime}{ }_{j}}$,

2) $b_{i i}=m_{a_{i} a^{\prime}{ }_{i}}$,

3) $\delta<m_{a_{i} a^{\prime}{ }_{j}}$,

4) $\delta<m_{a_{i} a^{\prime}{ }_{i}}$.

Besides the general statement of our results we will work only with the case $\kappa=\omega$ in which we drop the $\kappa^{+}=\omega_{1}$ in the definitions. Most of the comments have obvious generalizations to the general $\kappa$ case. One can also multiply the definitions of the related properties, e.g., one can consider matrices of matrices etc. There is a considerable group of such properties which hold for our generic construction of section 3, and which can be extracted from the forcing proof.

Note that the behaviour of the matrices $M$ defined in Definition 1 . on the diagonal is irrelevant. One should also note that symmetric matrices are just colorings of pairs $c:[\lambda]^{2} \rightarrow \kappa^{+}$. Thus, we are really interested in obtaining some values (depending on cases 1-4 of Definition 1) on $c^{\prime \prime}\left[a_{\xi} \otimes a_{\eta}\right]$ for some $\xi<\eta<\kappa^{+}$ and any family of disjoint $\nu$-tuples $a_{\xi}$ where $a_{\xi} \otimes a_{\eta}=\left\{\{\alpha, \beta\}: \alpha \in a_{\xi}, \beta \in a_{\eta}\right\}$. In order to agree with the existing terminology, the colorings $c:[\lambda]^{2} \rightarrow \kappa^{+}$ which give (diagonally) $\kappa^{+}$-strongly unbounded $\lambda \times \lambda$ matrices will be called (diagonally) $\kappa^{+}$-strongly unbounded functions on $\lambda$. If a property of a matrix, from Definition 1. holds only for $\nu=1$, we drop the word "strongly", e.g. we 
will talk about of a function which is universal or unbounded. In the case of the unbounded property this terminology agrees with the one of [T5], section 14, but disagrees with the terminology of [EGKTT] section 5 .

The case $\lambda=\omega_{1}$ is nowadays well investigated. Strongly unbounded functions on $\omega_{1}$ are trivial i.e., $\max (i, j)$ serves. A breakthrough was obtained in [T2] where Todorcevic constructed first $\omega_{1} \times \omega_{1}$ universal matrix. Actually, as known today, this matrix is $\omega_{1} \times \omega_{1}$ diagonally strongly universal (see [Be], [T5]; however the property was introduced in [S2] for a coloring motivated by [T2]). As noted in [SS2] there is no ZFC $\omega_{1} \times \omega_{1}$ strongly universal matrix: arbitrary behaviour of the entries of the matrix on uncountable collections for $n$-tuples would give the c.c.c. of a forcing which would add an uncountable subcollection with fixed behaviour; in particular $\mathrm{MA}+\neg \mathrm{CH}$ implies that there is no such matrix. On the other hand the $\mathrm{CH}$ or forcing with finite conditions gives a strongly universal matrix.

For $\lambda=\omega_{2}, \kappa=\omega$, to get even the weakest objects of our consideration we need to assume the negation of the Chang's Conjecture (CC) (see [Ka]). Actually $\neg \mathrm{CC}$ is equivalent to the existence of a strongly unbounded function on $\omega_{2}$ and to the existence of an unbounded function on $\omega_{2}$ (see [T3], [T5]). To get any matrix with some universal property we need to assume the negation of the continuum hypothesis $(\mathrm{CH})$ (Use the Erdös-Rado theorem for some partition of $\omega_{1}$ colors into $\omega$ disjoint groups). As we note in general in section 2 , the existence of a $\kappa^{+}$-strongly unbounded function on $\lambda$ implies the existence of a $\kappa^{+}$-c.c. forcing which adds a $\lambda$ by $\lambda \kappa^{+}$-strongly universal matrix. However we do not know the answer to the following:

Question 2. Is $\neg C C+\neg C H$ equivalent to the existence of (diagonally, strongly) universal matrix on $\omega_{2}$ ? Equivalently (by [T3], see [T5] section 14.), does $\neg \mathrm{CH}$ and the existence of a strongly unbounded function on $\omega_{2}$ imply the existence of (diagonally, strongly) universal matrix on $\omega_{2}$ ?

If we skip the word "diagonal" but keep the word "strongly" then as in the case of $\lambda=\omega_{1}$, for example MA $+\neg \mathrm{CH}$ implies the nonexistence of such a function.

The subject of this paper is the case of $\lambda>\omega_{2}$ and its comparison with the previous cases. Our main positive result which is a consequence of Theorem 33, is the following:

Theorem 3. Let $\kappa$ be a regular cardinal and $\lambda$ a cardinal such that $\kappa^{+++} \leq \lambda$. It is consistent that there is a $\lambda$ by $\lambda \kappa^{+}$-strongly universal matrix and so it is consistent that there is $\kappa^{+}$-strongly unbounded function on $\lambda$.

However we obtain a series of results which show a very different behaviour already of unbounded functions on $\lambda$ for $\lambda>\omega_{2}$ compared to the case of $\lambda=\omega_{2}$. We just mention the case $\kappa=\omega$ in the following summary, but similar results hold for other values of $\kappa$.

Theorem 4. Suppose $\lambda>\omega_{2}$.

a) If there is a strongly unbounded function on $\lambda$, then $\lambda \geq 2^{\omega}$. 
b) Suppose CH. There is no iteration of a $\sigma$-closed forcing followed by a c.c.c. forcing which adds a strongly unbounded function on $\lambda$.

c) It is consistent that there is an unbounded function on $\lambda$ but there is no strongly unbounded function on $\lambda$.

d) It is consistent that the model theoretic transfer principle $\left(\lambda, \omega_{1}\right) \rightarrow\left(\omega_{1}, \omega\right)$ is false but there is no strongly unbounded function on $\lambda$.

Proof.

(a) is a special case of Theorem 14 .

(b) follows from (a) as a $\sigma$-closed forcing preserves $\mathrm{CH}$ and a c.c.c. forcing cannot add a strongly unbounded function on $\lambda$ (every function into $\omega_{1}$ in the extension is bounded by one in the ground model).

To prove $(c)$, following $[\mathrm{R}]$, if $T$ is a Kurepa tree with $\lambda$-many branches numbered as $\left\{b_{\alpha}: \alpha<\lambda\right\}$, then $f(\alpha, \beta)=\min \left\{\xi<\omega_{1}: b_{\alpha}(\xi) \neq b_{\beta}(\xi)\right\}$ has the property that the image of all pairs of any uncountable set is uncountable (this function is also diagonally unbounded). It is well-known that the existence of such trees is consistent with $\mathrm{CH}$, thus (a) implies $(c)$.

To prove $(d)$, note as in $(c)$ that it is consistent that there is a Kurepa tree with $\lambda$-many branches (thus $\left(\lambda, \omega_{1}\right) \rightarrow\left(\omega_{1}, \omega\right)$ fails) but there is no strongly unbounded function on $\lambda$.

This should be contrasted with the fact that for $\lambda=\omega_{2}$ all of the above conditions are false. The standard unbounded functions like $\rho$ or the morass coloring (see $[\mathrm{M}]$ ) are compatible with $\mathrm{CH}$. They also can be added over any model of $\mathrm{CH}$ by a $\sigma$-closed forcing as morasses or the square can be added by such forcings. Finally the conditions from $(c),(d)$ are equivalent to the existence of a strongly unbounded function on $\omega_{2}$ (see [T5] section 14).

The condition (b) also shows that we cannot hope for adding a strongly unbounded functions on larger cardinals, the usual way i.e., by an iteration of the type mentioned in this theorem. Our generic construction uses the method of forcing with side conditions in semimorasses. A similar method of forcing with side conditions in morasses has been successfully introduced in [Ksz3] to solve a problem of A. Hajnal, the situation where also an iteration $\sigma$-closed forcing followed by a c.c.c. forcing cannot be used. Both of these methods can be considered as versions of Todorcevic's method of forcing with matrices of models as side-conditions (see [T1]).

1.4. Towards applications outside combinatorics. The first place to look for applications of our results outside combinatorics is to look at generalizations of already existing results i.e., [BS], [BSp], [EGKTT], [Kj], [Ksz2], [Ksz3], [Ksz4], $[\mathrm{SSp}],[\mathrm{SS} 1],[\mathrm{SS} 2],[\mathrm{T} 3],[\mathrm{Ve}],[\mathrm{Z}]$. We found two immediate applications yielding new consistency results. 
Theorem 5. It is consistent for every cardinal $\lambda$ that the square of the sequential fan with $\lambda$-many spines times $\omega_{1}$ with the usual order topology is a normal topological space. ${ }^{8}$

Proof. Immediate applications of [EGKTT] 5.2. and Theorem 3 and Fact 12 which can be used to obtain a model with an unbounded function on $\lambda$ that satisfies $\mathrm{MA}_{\lambda}$.

Theorem 6. Suppose that $\lambda \geq \kappa^{++}$and $\kappa$ is a regular cardinal. It is consistent that there is is a full-angled ${ }^{9}$ bilinear space of dimension $\lambda$ over any field of cardinality $\kappa$

Proof. Let $\left\{k_{\alpha}: \alpha<\kappa\right\}$ be an enumeration of a field $K$ of cardinality $\kappa$. Define the bilinear form to be $\psi\left(e_{\alpha}, e_{\beta}\right)=k_{f(\alpha, \beta)}$ where $f$ is a $\lambda$ by $\lambda \kappa^{+}$-strongly universal matrix and $\left\{e_{\alpha}: \alpha<\lambda\right\}$ a basis of the vector space $V$. One easily proves that $\psi$ is full-angled.

We also note that the unbounded function may be used as an elegant axiomatization which implies known results. Below, using the existence of unbounded functions we conclude the result of $[\mathrm{Z}]$.

Theorem 7. Assume $M A_{\lambda}$ and the existence of a strongly unbounded function on $\lambda$, then there is a family of functions $\left\{f_{\alpha}: \alpha<\lambda\right\} \subseteq \omega^{\omega_{1}}$ which is almost disjoint i.e., $\left\{\xi \in \omega_{1}: f_{\alpha}(\xi)=f_{\beta}(\xi)\right\}$ is finite for every distinct $\alpha, \beta \in \lambda$.

Proof. Let $f:[\lambda]^{2} \rightarrow \omega_{1}$ be a strongly unbounded function on $\lambda$. Let $P$ be forcing consisting of conditions of the form $p=\left(a_{p}, b_{p},\left\{f_{p}^{\alpha}: \alpha \in a_{p}\right\}\right)$ where:

1) $a_{p} \in[\lambda]^{<\omega}, b_{p} \in\left[\omega_{1}\right]^{<\omega}, f_{p}^{\alpha}: b_{p} \rightarrow \omega$,

2) $f_{\alpha}(\xi) \neq f_{\beta}(\xi)$ for $\xi>f(\alpha, \beta)$. We define $p \leq q$ if and only if

3) $a_{p} \supseteq a_{q}, b_{p} \supseteq b_{q}$ and $f_{p}^{\alpha} \mid b_{q}=f_{q}^{\alpha}$ for $\alpha \in a_{q}$,

4) whenever $\alpha, \beta \in a_{q}$ and $\xi \in b_{p}-b_{q}$, then $f_{p}^{\alpha}(\xi) \neq f_{p}^{\beta}(\xi)$.

Simple density arguments show that it is enough to prove that $P$ satisfies the c.c.c. For this we choose an uncountable sequence $\left(p_{\xi}: \xi<\omega_{1}\right)$ of conditions of $P$. We may w.l.o.g. assume that $a_{p_{\xi}}$ 's form a $\Delta$-system with root $\Delta$ and that $b_{p_{\xi}}$ 's form a "head-tail-tail" $\Delta$-system with root $\Gamma$ and that the conditions are isomorphic and agree on $\Delta \times \Gamma$. Let $p, q$ be any two conditions like above which moreover satisfy $f(\alpha, \beta)>\Gamma$ for any $\alpha \in a_{p}-a_{q}$ and $\beta \in a_{q}-a_{p}$ and

\footnotetext{
${ }^{8}$ This result is related to the question whether Lašnev spaces (images of metric spaces under closed mappings) have normal $\Sigma$-products. It was proved in [EGKTT] that this generalization of the theorem of Gulko and Rudin about normality of $\Sigma$-products of metric spaces is consistently false. Actually the consistent non-normality of $S\left(\omega_{2}\right)^{2} \times \omega_{1}$ was used for this purpose. The search for ZFC counterexamples naturally faced spaces of the form $S(\lambda)^{2} \times \omega_{1}$ and the above result is a part of it. On the other hand it is open weather $S\left(2^{\omega_{1}}\right)^{2} \times \omega_{1}$ is non-normal, and so this space could be a ZFC counterexample.

${ }^{9} \mathrm{~A}$ bilinear space i.e. a vector space with a symmetric bilinear form $(V, \Psi)$ is said to be full-angled if it assumes all values (from the field) on any set of vectors (see [BSp], §3, we are generalizing the definition to fit our generalization) of cardinality $\kappa^{+}$
} 
$f^{\prime \prime}[\Delta]^{2} \cap\left(b_{q}-\Gamma \cup b_{p}-\Gamma\right)=\emptyset$. This can be accomplished by the fact that $f$ is strongly unbounded on $\lambda$.

Define an amalgamation $r$ of $p$ and $q$ as $r=\left(a_{p} \cup a_{q}, b_{p} \cup b_{q},\left\{f_{r}^{\alpha}: \alpha \in a_{r}\right\}\right)$ where

- $f_{r}^{\alpha}=f_{p}^{\alpha} \cup h^{\alpha}$ for $\alpha \in a_{p}-a_{q}$,

- $f_{r}^{\alpha}=f_{q}^{\alpha} \cup g^{\alpha}$ for $\alpha \in a_{q}-a_{p}$,

- $f_{r}^{\alpha}=f_{p}^{\alpha} \cup f_{q}^{\alpha}$ for $\alpha \in a_{p} \cap a_{q}$.

Note that $h^{\alpha}$ 's and $g^{\alpha}$ 's can be easily chosen (just take all distinct and new values) so that 2) and 4) are satisfied if $\{\alpha, \beta\} \in\left[a_{p}\right]^{2} \cup\left[a_{q}\right]^{2}-[\Delta]^{2}$ and 2) is satisfied if $\{\alpha, \beta\} \in\left[a_{p}-\Delta\right]^{2} \otimes\left[a_{q}-\Delta\right]^{2}$ and $\xi \notin \Gamma$. 2) for $\{\alpha, \beta\} \in\left[a_{p}-\Delta\right]^{2} \otimes$ $\left[a_{q}-\Delta\right]^{2}$ and $\xi \in \Gamma$ does not need to be checked by the assumption about $p$ and $q$ which follows from the unboundedness of $f$. So we are left with the case $\alpha, \beta \in \Delta$ to be checked. Now 2) is trivial by 2) for $p$ and $q$ and 4) follows from 2 ) for $p$ and $q$ and the the assumption $f^{\prime \prime}[\Delta]^{2} \cap\left(b_{q}-\Gamma \cup b_{p}-\Gamma\right)=\emptyset$.

Many other constructions of the above listed papers apparently depend on properties of colorings which cannot be generalized above $\omega_{2}$. Consider the following weakening of the $\Delta$-property of [BS] quite often sufficient to obtain the applications.

Definition 8. $F:\left[\omega_{2}\right]^{2} \rightarrow\left[\omega_{2}\right] \leq \omega$ is said to have the weak $\Delta$-property if and only if whenever $\left(a_{\xi}: \xi<\omega_{1}\right)$ is a family finite subsets of $\omega_{2}$, then there are distinct $\xi, \eta \in \omega_{2}$ such that

$$
a_{\xi} \cap a_{\eta} \cap \min (\alpha, \beta) \subseteq f(\alpha, \beta)
$$

for all $\alpha \in a_{\xi}-a_{\eta}$ and $\beta \in a_{\eta}-a_{\xi}$.

It is implicitly proved in [T3] and [T5] that the existence of a function with the weak $\Delta$-property is equivalent to the existence a strongly unbounded function on $\omega_{2}$. It is also easy to see that there cannot be any function with the weak $\Delta$-property on cardinals above $\omega_{2}$.

Finally, we note that the strongly unbounded functions alone apparently do not advance the matter of most prominent problems in the area, the generalization of the result from [BS], i.e., a generic construction of a superatomic Boolean algebra of hight $\omega_{3}$ and width $\omega$, and the generalization of the result from [T3], i.e., a generic construction of a coloring $c: \omega_{3} \times \omega_{3} \rightarrow \omega$ nonconstant on product of any two infinite sets.

\section{The universal and the unboundedness properties}

2.1. The unboundedness property and c.c.c. forcings. In this section we work with $\kappa=\omega$, but all results have natural generalizations to higher cardinals.

Definition 9. A strongly unbounded function $f:[\lambda]^{2} \rightarrow \omega_{1}$ is called c.c.c. indestructible on $\lambda$ if and only if it remains strongly unbounded in every generic extension obtained by a c.c.c. forcing. 
Fact 10. $f:[\lambda]^{2} \rightarrow \omega_{1}$ is c.c.c. indestructible strongly unbounded if for every uncountable pairwise disjoint family $\left\{a_{\xi}: \xi<\omega_{1}\right\}$ of finite subsets of $\lambda$ and every countable ordinal $\delta$ there is an uncountable $A \subseteq \omega_{1}$ such that $f^{\prime \prime}\left[a_{\xi} \otimes a_{\eta}\right]>\delta$ for every $\xi, \eta \in A$.

Proof. Suppose $f$ satisfies the property from the fact and let $P$ be a c.c.c. forcing notion. Suppose that $f$ is not strongly unbounded in $V^{P}$. It must be witnessed by a countable ordinal $\delta$ and an uncountable pairwise disjoint family $\left\{a_{\xi}: \xi<\right.$ $\left.\omega_{1}\right\}$ of finite subsets of $\lambda$. Let $p \in P$ decide $\delta$ and let $p_{\xi}$ be a condition of $P$ below $p$ which decides $a_{\xi}$. Let $A$ be as in the property stated in the fact. Then $\left\{p_{\xi}: \xi \in A\right\}$ must form an antichain which contradicts the c.c.c. of $P$.

It is proved in [T5] corollary 14.7, that if there is a strongly unbounded function $f:\left[\omega_{2}\right]^{2} \rightarrow \omega_{1}$, then there is one which satisfies the property from the fact, in particular one that is c.c.c. indestructible. We do not know if the same is true for $\lambda>\omega_{2}$. Note that two well-known unbounded functions on $\omega_{2}, \rho$ ( see $[\mathrm{Be}]$ ) and the morass coloring (first investigated in [M], see also [EGKTT]) are both subadditive (see Section 14 of [T5]) and hence they are c.c.c. indestructible. Our generic constructions of section 3.3. provide apparently first examples of strongly unbounded functions which are not c.c.c. indestructible (see remark 34). It is not difficult to make a strongly unbounded function a c.c.c. indestructible one as seen in the following results.

Lemma 11. Suppose $\mathcal{A}$ is a family of disjoint finite subsets of $\lambda$. Suppose $f$ : $[\lambda]^{2} \rightarrow \omega_{1}$ is strongly unbounded. Suppose $\delta<\omega_{1}$. Let $Q(\mathcal{A}, \delta)$ be the forcing consisting of finite subcollections $A$ of $\mathcal{A}$ such that for every distinct $a, b \in A$ we have $f^{\prime \prime}[a \otimes b]>\delta$. Then $Q$ satisfies the c.c.c. and $Q \Vdash \mathscr{f}$ is unbounded.

Proof. Let $\left\{A_{\xi}: \xi<\omega_{1}\right\}$ be an uncountable sequence of elements of $Q$. For every $\xi<\omega_{1}$ define $a_{\xi}=\bigcup A_{\xi}$. $a_{\xi}$ 's are finite as $A_{\xi}$ 's were finite families of finite sets. We may w.l.o.g. assume that $a_{\xi}$ 's form a $\Delta$-system with root $\Delta$ and that $A_{\xi} \cap \wp(\Delta)=A_{\xi^{\prime}} \cap \wp(\Delta)$ for every $\xi, \xi^{\prime}<\omega_{1}$. Let $b_{\xi}=a_{\xi}-\Delta$. Now apply the unboundedness of $f$, to $\delta$ and $b_{\xi}$ 's obtaining $\xi_{1}, \xi_{2}<\omega_{1}$ such that for all $\alpha \in b_{\xi_{1}}$ and $\beta \in b_{\xi_{2}}$ we have $f(\alpha, \beta)>\delta$. It is easy to see that this is enough for the compatibility of $A_{\xi_{1}}$ and $A_{\xi_{2}}$.

Now suppose that $f$ is no longer unbounded in $V^{Q}$. Let $\left\{A_{\xi}: \xi<\omega_{1}\right\}$ and $\left(F_{\xi}: \xi<\omega_{1}\right)$ and $\left(\dot{F}_{\xi}: \xi<\omega_{1}\right)$ be such that $Q$ forces that $\left(\dot{F}_{\xi}: \xi<\omega_{1}\right)$ and $\delta<\omega_{1}$ witness the failure of the unboundedness of $f$ and

$$
A_{\xi} \Vdash \dot{F}_{\xi}=\check{F}_{\xi}
$$

Perform similar construction as in the proof of the c.c.c., but now define

$$
a_{\xi}=\bigcup A_{\xi} \cup F_{\xi}
$$

By disjointness of $\left(F_{\xi}: \xi<\omega_{1}\right)$ it can be seen that similar argument as in the proof of the c.c.c give us compatible $A_{\xi_{1}}$ and $A_{\xi_{2}}$ such that for $\alpha \in F_{\xi_{1}}, \beta \in F_{\xi_{2}}$ we have $f(\alpha, \beta)>\delta$ contradicting the choice of $\left(\dot{F}_{\xi}: \xi<\omega_{1}\right)$. 
Fact 12. Suppose $f:[\lambda]^{2} \rightarrow \omega_{1}$ is strongly unbounded on $\lambda$. There is a c.c.c. forcing notion $Q$ such that in $V^{Q}$ the function $f$ is c.c.c. indestructible.

Proof. $Q$ is an iteration of length $\lambda^{+}$with finite supports of the forcings $Q(\mathcal{A}, \delta)$ of Lemma 11, so that every $\delta \in \omega_{1}$ and every uncountable family of disjoint finite sets of $\lambda$ in some intermediate model are taken care of. Note that if $Q$ is c.c.c, it works. Indeed in $V^{Q}$ for every family of disjoint finite sets $\mathcal{A}$ in $\lambda$ and $\delta<\omega_{1}$ there is an uncountable family $\mathcal{B} \subseteq \mathcal{A}$ such that for every distinct $a, b \in \mathcal{B}$ we have $f^{\prime \prime}[a \otimes b]>\delta$.

So now, let's see that $Q$ is c.c.c. For this it is enough to prove by induction on $\alpha<\lambda^{+}$that any iteration of length $\alpha$ of forcings like $Q(\mathcal{A}, \delta)$ satisfies the c.c.c. This follows from the fact that finite support iteration of forcings satisfying the c.c.c. satisfy the c.c.c and the previous lemma, provided we know that the iteration preserves the unboundedness of $f$. We prove it by induction as well. The successor stage is taken care of by the previous lemma. The limit stage of cofinality different than $\omega_{1}$ is trivial. Now consider $\left(Q_{\beta}, \dot{Q}^{\beta}\right)_{\beta<\alpha}$ for $\alpha$ of cofinality $\omega_{1}$, and assume that you know that in each $V^{P_{\beta}} f$ is unbounded. Let $\delta<\omega_{1},\left\{q_{\xi}: \xi<\omega_{1}\right\},\left(G_{\xi}: \xi<\omega_{1}\right),\left(F_{\xi}: \xi<\omega_{1}\right)$ and $\left(\dot{F}_{\xi}: \xi<\omega_{1}\right)$ be such that $Q_{\alpha}$ forces that $\left(\dot{F}_{\xi}: \xi<\omega_{1}\right)$ and $\delta$ witness the failure of the unboundedness of $f$ and

$$
q_{\xi} \Vdash \dot{F}_{\xi}=\check{F}_{\xi}
$$

and $\operatorname{supp}\left(q_{\xi}\right)=G_{\xi}$. We may w.l.o.g. assume that $\left(G_{\xi}: \xi<\omega_{1}\right)$ form a $\Delta$-system with the root below $\beta_{0}<\alpha$.

Now work in $V^{Q_{\beta_{0}}}$. By the inductive assumption about the c.c.c. of $Q_{\beta_{0}}$ we know that for an uncountable set $X \subseteq \omega_{1}$ and $\xi \in X$ condition $q_{\xi} \mid \beta_{0}$ is in the generic filter raising to the extension $V^{P_{\beta_{0}}}$. Apply the unboundedness of $f$ in $V^{P_{\beta_{0}}}$ to $\left(F_{\xi}: \xi \in X\right)$ and $\delta$ obtaining $\xi_{1}, \xi_{2} \in X$ such that $f^{\prime \prime}\left[F_{\xi_{1}} \otimes F_{\xi_{2}}\right]>\delta$. Now note that since $G_{\xi}$ 's formed a $\Delta$-system with the root below $\beta_{0}$ and $q_{\xi_{1}} \mid \beta_{0}$ and $q_{\xi_{2}} \mid \beta_{0}$ were compatible, we have that $q_{\xi_{1}}$ and $q_{\xi_{2}}$ are compatible, which gives the required contradiction.

Fact 13. (Implicitly [S2], section 4) Suppose there is an unbounded function $f:[\lambda]^{2} \rightarrow \omega_{1}$. Then, there is a c.c.c. forcing $P$ such that in $V^{P}$ there is $a \lambda \times \lambda$ strongly universal matrix.

Proof. Let $f:[\lambda]^{2} \rightarrow \omega_{1}$ be strongly unbounded. Define a forcing $P$ to have conditions of the form $\left(a_{p},\left(m_{p}(\alpha, \beta):\{\alpha, \beta\} \in\left[a_{p}\right]^{2}\right)\right)$ where $a_{p}$ is a finite subset of $\lambda$ and $m_{p}(\alpha, \beta)<f(\alpha, \beta)$ 's are countable ordinals. The order is the inverse inclusion.

We claim that $\lambda$ is preserved by $P$ and that

$$
\bigcup\left\{m_{p}(\alpha, \beta):\{\alpha, \beta\} \in\left[a_{p}\right]^{2}, p \in G\right\}
$$

is a strongly universal matrix, where $G$ is a $P$-generic over $V$. We will actually prove that $P$ satisfies the c.c.c. and the strong universal property in one 
argument. Let $\left(p_{\xi}: \xi<\omega_{1}\right)$ be conditions of $P$ and let $\left(\dot{F}_{\xi}: \xi<\omega_{1}\right)$ be $P$ names for disjoint $n$-element subsets of $\lambda$ and let $B: n \times n \rightarrow \omega_{1}$ be an $n$ by $n$ matrix. By extending the conditions, we may w.l.o.g. assume that there are finite sets $\left(F_{\xi}: \xi<\omega_{1}\right\}$, with $F_{\xi}=\left\{a_{\xi}^{i}: i<n\right\}$ such that $p_{\xi} \|-\dot{F}_{\xi}=\check{F}_{\xi}$ and that $a_{p_{\xi}}$ 's form a $\Delta$-system of sets with its root $\Delta$ and that $F_{\xi} \subseteq a_{p_{\xi}}$. Note that by the disjointness of $F_{\xi}$ 's one can assume that they are disjoint from $\Delta$. Further, by the fact that $m_{p}(\alpha, \beta)<f(\alpha, \beta)$ holds, we can assume that all the conditions agree on the root. Finally using the unboundedness property of $f$ we can find two distinct ordinals $\xi<\eta<\omega_{1}$ such that $f(\alpha, \beta)>\max (\operatorname{ran}(B))$ for all $\alpha \in a_{p_{\xi}}-a_{p_{\eta}}$ and $\beta \in a_{p_{\xi}}-a_{p_{\eta}}$. Now it is easy to construct an amalgamation $r$ of $p_{\xi}$ and $p_{\eta}$ defining $m_{r}$ on $\left(a_{p_{\xi}}-a_{p_{\eta}}\right) \otimes\left(a_{p_{\xi}}-a_{p_{\eta}}\right)$ arbitrarily as long as it has values below $f(\alpha, \beta)>\max (\operatorname{ran}(B))$. In particular one can define it so that $m_{r}\left(\alpha_{\xi}^{i}, \alpha_{\eta}^{j}\right)=B(i, j)$ for any $i, j<n$. This completes the proof of the c.c.c. for $P$ and the strongly universal property.

\subsection{It is hard to get strongly unbounded functions on bigger cardi-} nals.

Theorem 14. If $2^{\kappa}<\lambda$ and $\lambda>\kappa^{++}$, then there is no $\kappa^{+}$-strongly unbounded function on $\lambda$.

Proof. Suppose $2^{\kappa}<\lambda$ and $\lambda>\kappa^{++}$, and that $f:[\lambda]^{2} \rightarrow \kappa^{+}$. We will show that $f$ is not strongly unbounded. We need an elementary submodel $M$ of the structure $H(\theta)$ for $\theta$ big enough such that $f, \lambda \in M,[M]^{\kappa} \subseteq M$ and there is an ordinal $\beta \in \lambda-M$ such that $\operatorname{cf}(\beta)=\kappa^{++}$and $M \cap \lambda$ is unbounded in $\beta$. For this one can take $\zeta=\max \left(\kappa^{++}, 2^{\kappa}\right)<\lambda$ note that $\zeta^{\kappa}=\zeta$ and so one can construct a chain of models closed under $\kappa$-sequences of cardinality $\zeta$ so that $M_{\xi} \cap \zeta^{+}<M_{\eta} \cap \zeta^{+}$for $\xi<\eta<\kappa^{++}$. The union works together with its supremum in $\zeta^{+} \leq \lambda$.

Consider $F: \beta \rightarrow \kappa^{+}$defined by $F(\alpha)=f(\alpha, \beta)$ for $\alpha<\beta$. Note that as $\operatorname{cf}(\beta)=\kappa^{++}$we have $\delta \in \kappa^{+}$as well as a set $A \subseteq M \cap \beta$ of cardinality $\kappa^{++}$ unbounded in $\beta$ such that $F(\alpha)=\delta$ for each $\alpha \in A$. Now construct by induction two sequences $\left\{\alpha_{\xi}: \xi<\kappa^{+}\right\} \subseteq A$ and $\left\{\beta_{\xi}: \xi<\kappa^{+}\right\} \subseteq M \cap \alpha$ such that

i) For every $\xi<\eta<\kappa^{+}$we have $\alpha_{\xi}<\beta_{\xi}<\alpha_{\eta}<\beta_{\eta}<\beta$.

ii) For every $\xi<\eta<\kappa^{+}$we have $f\left(\alpha_{\xi}, \beta_{\eta}\right)=\delta$.

Suppose we are done below $\eta$. Now as $[M]^{\kappa} \subseteq M$, we have $\left\{\alpha_{\xi}: \xi<\eta\right\} \in M$. Choose as $\alpha_{\eta}$ any element of $A$ above $\sup \left(\left\{\beta_{\xi}: \xi<\eta\right\}\right)$. Clearly, in $H(\theta)$ there is an ordinal $\beta$ such that $\alpha_{\eta}<\beta<\min (M-\beta)$ such that $f\left(\alpha_{\xi}, \beta\right)=\delta$ for all $\xi<\eta$. By the elementarity, in $M$, choose such an ordinal and call it $\beta_{\eta}$. Now note that $\left\{\left\{\alpha_{\xi}, \beta_{\xi}\right\}: \xi<\kappa^{+}\right\}$contradicts the unboundedness property, and so $f$ is not unbounded.

\section{The generic construction}

3.1. Semimorasses. In [Ksz1], we introduced some generalization of Velleman's simplified morasses (see [Ve1], [Ve2]). A natural way of looking at morasses 
or semimorasses is to see them as families similar to $\left\{M \cap \omega_{2}: M \prec H\left(\omega_{3}\right)\right\}$ with some extra coherence properties (which actually make it impossible for a morass to include a club set unlike the set above). To follow our notation we quote below this definition in the special case of a successor cardinal.

Definition 15. Let $\kappa$ be a regular cardinal. A simplified $\left(\kappa^{+}, \lambda\right)$-semimorass is a family $\mathcal{F} \subseteq[\lambda]^{\kappa}$ which satisfies the following conditions.

1) $\mathcal{F}$ is well-founded with respect to inclusion.

2) $\mathcal{F}$ is locally small i.e. $\forall X \in \mathcal{F}|\mathcal{F}| X \mid \leq \kappa$ where $\mathcal{F} \mid X=\{Y \in \mathcal{F}: Y \subset X\}$

3) $\mathcal{F}$ is homogeneous i.e. if $X, Y \in \mathcal{F}, \operatorname{rank}(X)=\operatorname{rank}(Y)=\alpha$, then $X, Y$ have the same order type (denoted $\theta_{\alpha}$ ) and if $f_{X Y}$ is the unique order preserving mapping from $X$ onto $Y$, then $\mathcal{F} \mid Y=\left\{f^{\prime \prime}(Z): Z \in \mathcal{F} \mid X\right\}$.

4) $\mathcal{F}$ is directed i.e. $\forall X, Y \in \mathcal{F} \exists Z \in \mathcal{F}$ s.t. $X, Y \subseteq Z$

5) $\mathcal{F}$ is locally semi directed, i.e.,

a) $\mathcal{F} \mid X$ is directed or

b) $\exists X_{1}, X_{2} \in \mathcal{F}$ s.t. $\operatorname{rank}\left(X_{1}\right)=\operatorname{rank}\left(X_{2}\right) \& X=X_{1} * X_{2}$ where $X=$ $X_{1} * X_{2}$ means that $X=X_{1} \cup X_{2}, \mathcal{F}|X=\mathcal{F}| X_{1} \cup \mathcal{F} \mid X_{2} \cup$ $\left\{X_{1}, X_{2}\right\}$ and for every $\alpha \in X_{1} \cap X_{2}$ we have

$$
\operatorname{ordtp}\left(X_{1} \cap \alpha\right)=\operatorname{ordtp}\left(X_{2} \cap \alpha\right)
$$

6) $\mathcal{F}$ covers $\kappa^{+}$i.e. $\bigcup \mathcal{F}=\kappa^{+}$.

Semimorasses have some properties analogous to the properties of Velleman's morasses.

Lemma 16 (The main lemma for semimorasses.). ([Ksz1]) Let $\mathcal{F}$ be $a\left(\kappa^{+}, \lambda\right)$ semimorass. Let $X, Y \in \mathcal{F}, \operatorname{rank}(X)=\operatorname{rank}(Y), \alpha \in X \cap Y$, then $\operatorname{ordtp}(X \cap$ $\alpha)=\operatorname{ordtp}(Y \cap \alpha)$.

Lemma 17 (Density lemma for semimorasses). ([Ksz1]) Let $\mathcal{F}$ be $a\left(\kappa^{+}, \lambda\right)$ semimorass. Then the following conditions are satisfied:

(a) $\forall Y \in \mathcal{F} \forall X \in(\mathcal{F} \mid Y) \forall \operatorname{rank}(X)<\alpha<\operatorname{rank}(Y) \exists Z \in \mathcal{F} \operatorname{rank}(Z)=\alpha$,

$$
X \subseteq Z \subseteq Y
$$

i.e. for example $\mathrm{ht}(\mathcal{F}) \leq \kappa$, since $\mathcal{F}$ is locally small.

(b) $\forall X \in \mathcal{F} \forall \alpha<\operatorname{ht}(\mathcal{F})[\operatorname{rank}(X)<\alpha \Rightarrow \exists Z \in \mathcal{F}(\operatorname{rank}(Z)=\alpha, X \subseteq Z)]$

In this paper we will assume that the semimorass in question which we will be denoting by $\mathcal{F}$ is a stationary coding set (see $[\mathrm{Zw}]$ ). This means that $\mathcal{F}$, is stationary subset of $[\lambda]^{\kappa}$ and there is a one-to-one function $c: \mathcal{F} \rightarrow \lambda$ such that

$$
\forall X, Y \in \mathcal{F} \quad X \subset Y \Rightarrow c(X) \in Y .
$$

The forcing proof of the consistency of the GCH below $\kappa^{+}$with the existence (for any regular $\kappa$ and $\lambda \geq \kappa^{+}$) of a $\left(\kappa^{+}, \lambda\right)$-semimorasses which are stationary coding sets which is based on a proof of Velleman from [V2] is published in [Ksz1] (Theorem 3, section 2). Let's note two simple facts about stationary coding sets in general which we learned from S. Todorcevic. 
Fact 18 (folklore). Suppose that $\mathcal{F} \subseteq[\lambda]^{\kappa}$ is a stationary coding set and $\mathcal{F} \in$ $M \prec H\left(\lambda^{+}\right),|M|=\kappa, M \cap \lambda \in \mathcal{F}$. If $X \in \mathcal{F}$ and $X \subset M$, then $X \in M$.

Proof. Suppose $X \in \mathcal{F}$ and $X \subset M$. As $\mathcal{F} \in M \prec H\left(\lambda^{+}\right)$, we have that $M$ thinks that $\mathcal{F}$ is a stationary coding set, so there is $c: \mathcal{F} \rightarrow \lambda$ witnessing this fact in $M$. In particular $\alpha=c(X) \in M \cap \lambda$, so $M$ knows $c^{-1}(\alpha)$, hence $X \in M \cap[\lambda]^{\kappa}$ as required.

Fact 19 (folklore). Suppose that $\mathcal{F} \in M \prec H\left(\lambda^{+}\right),|M|=\kappa, X=M \cap \lambda \in \mathcal{F}$. Then $\operatorname{rank}(X)=M \cap \kappa^{+}$.

Proof. As $\mathcal{F} \in M$ we have that $\kappa \in M$ and so $M \cap \kappa^{+} \in \kappa^{+}$. Put $\operatorname{rank}(X)=\delta$. If $\delta \in M$, then we would have in $M$ an element $Y$ of $\mathcal{F}$ of rank $\delta$ and this would give rise by homogeneity to an isomorphism between $\mathcal{F} \mid Y$ and $\mathcal{F} \mid X$ which would contradict well-foundedness of $\mathcal{F}$.

In $M$ there are all ordinals less than $\delta$ and so there are also elements of $\mathcal{F}$ of all ranks less than $\delta$. They are included in $M \cap \lambda=X$, so $\operatorname{rank}(X)$ is at least $\delta$.

The facts below are crucial in our method of forcing with side condition in semimorasses. The property below resembles the definition of Kurepa family (see [T5]). Actually a Kurepa family which is a stationary coding set (coexisting with $\mathrm{CH}$ ) would be sufficient for our applications, but we do not know if such a family can consistently exist.

Fact 20. Suppose that a $\left(\kappa^{+}, \lambda\right)$-semimorass $\mathcal{F}$ is a stationary coding set and $\mathcal{F} \in M \prec H\left(\lambda^{+}\right),|M|=\kappa, M \cap \lambda=X_{0} \in \mathcal{F}$. Let $Y \in \mathcal{F}, \operatorname{rank}(Y)<M \cap \kappa^{+}=$ $\delta$. Then there is $Z(Y) \in M$ such that

1) $Y \cap X_{0} \subseteq Z(Y)$.

2) $\operatorname{rank}(Z(Y))=\operatorname{rank}(Y)$.

Proof. By density lemma, find $Y^{\prime} \supseteq Y, Y^{\prime} \in \mathcal{F}$ such that $\operatorname{rank}\left(Y^{\prime}\right)=\operatorname{rank}\left(X_{0}\right)=$ $\delta$. Now use the isomorphism $f_{Y^{\prime} X_{0}}$ to find a copy $Z(Y)$ of $Y$ below $X_{0}$. Note that $Y \cap X_{0} \subseteq Y^{\prime} \cap X_{0}$ and $f_{Y^{\prime} X_{0}}$ is constant on $Y^{\prime} \cap X_{0}$ so $Y \cap X_{0}=Y \cap M \subseteq Z$. Now use Fact 18, to conclude that $Z(Y) \in \mathcal{F} \mid X_{0}$ implies $Z(Y) \in M$.

Fact 21. Suppose that a $\left(\kappa^{+}, \lambda\right)$-semimorass $\mathcal{F}$ is a stationary coding set and $\mathcal{F} \in M \prec H\left(\lambda^{+}\right),|M|=\kappa, M \cap \lambda=X_{0} \in \mathcal{F},[M]^{<\kappa} \subseteq M$. Let $A \in[\mathcal{F}]^{<\kappa}$, Then there is $Z \in M$ such that

$$
\bigcup\left\{X \cap M: X \in A, \operatorname{rank}(X)<M \cap \kappa^{+}\right\} \subseteq Z
$$

Proof. Consider sets $Z(Y)$ obtained from Fact 20, for $Y \in A$ such that $\operatorname{rank}(Y)<$ $M \cap \kappa^{+}$. Now as $[M]^{<\kappa} \subseteq M$ the set of all these $Z(Y) \mathrm{s}$ is in $M$ and hence its union as well. Put $Z$ to be this union. 
3.2. Side conditions in semimorasses. To use elements of a semimorass $\mathcal{F}$ as side conditions in the simplest case $\kappa=\omega$, means to use forcings $P$ whose conditions are of the form $(p, A)$ where $p$ is a finite condition of a natural forcing adding the structure in question and $A$ is a finite subset of $\mathcal{F}$. This is like using models as side conditions in the method of forcing with models as side conditions developed by S. Todorcevic. The order is given by the forcing order on the first coordinate and the inverse inclusion on the second coordinate. In addition we require the existence of some natural projections of $p$ onto the elements of $A$ as a part of the definition of the forcing notion.

In the case of forcings described above, special combinatorial properties of semimorasses allow us to perform many manouvers with ease as well as the definitions are simplified. This method seems equivalent to the variant of Todorcevic's method when one employs matrices of models (see [T1] section 4., for an example with detailed definitions). Instead of a more complicated forcing that adds a version of a semimorass and the structure in question "in one blow" we factor this forcing into one adding a semimorass (or we actually just assume the existence of it) and another simple forcing employing the semimorass. The price we need to pay for this convenience is that $P$ is not proper (unlike Todorcevic's forcings, ) but only $\mathcal{F}$-proper, i.e., There is a club $\mathcal{C} \subseteq[\lambda]^{\omega}$ such that for models $M \prec H\left(\lambda^{+}\right)$such that $M \in \mathcal{F} \cap \mathcal{C}$ and $p \in P \cap M$, there are $(P, M)$-generic conditions stronger than $p$. As $\mathcal{F}$ may be assumed to be stationary, $\mathcal{F}$-properness implies the preservation of $\omega_{1}$ (proof like for proper forcings, see [B]). The preservation of bigger cardinals follows from the $\omega_{2}$-chain condition. Note that it is no limitation in the applications that one seeks here, i.e., consistent existence of structures of sizes bigger than $\omega_{1}$.

One can actually generalize this method to higher cardinals where the working part of the condition has size $<\kappa$ and the collection of side conditions is also of size less than $\kappa$. First let's define some simple generalizations related to properness.

Definition 22. Suppose $\mathcal{F} \subseteq[\lambda]^{\kappa}$. We say that a forcing notion $P$ is $\mathcal{F}$-proper on the level of $\kappa$ (we skip the phrase "on the level of $\kappa$ " if $\kappa=\omega$ ) if and only if there is $\theta>\left(2^{|P|}\right)^{+}$and a club set $\mathcal{C} \subseteq[H(\theta)]^{\kappa}$ such that whenever $p \in M \in \mathcal{C}$ and $M \cap \lambda \in \mathcal{F}$ then there is a $(P, M)$-generic $p_{0} \leq p$, i.e., $D \cap M$ is predense below $p_{0}$ for every $D \in M$ which is dense in $P$.

Fact 23. Suppose $\mathcal{F} \subseteq[\lambda]^{\kappa}$ is a stationary set and $P$ is an $\mathcal{F}$-proper on the level of $\kappa$ forcing notion, then $P$ preserves $\kappa^{+}$.

Proof. The proof is a straightforward version of Shelah's paradigmatic proof of preservation of $\omega_{1}$ by proper forcings (see [S1] or [B]).

The following definition and lemmas are formulations of well-known techniques (originated in Shelah's use of elementary submodels in forcing) and will simplify our further arguments. 
Definition 24. Let $P$ be a notion of forcing, and $q \in P$. Suppose $M \prec H(\theta)$ and $P, \pi_{1}, \ldots, \pi_{k} \in M$. We say that a formula $\phi\left(x_{0}, x_{1}, \ldots, x_{k}\right)$ well reflects $q$ in $\left(M ; \pi_{1}, \ldots, \pi_{k}\right)$ whenever the following are satisfied:

i) $\phi\left(q, \pi_{1}, \ldots, \pi_{k}\right)$ holds in $H(\theta)$

ii) whenever $s \in M$ is such that $\phi\left(s, \pi_{1}, \ldots, \pi_{k}\right)$ holds in $M$, then $q$ and $s$ are compatible.

Definition 25. Suppose that $\mathcal{F} \subseteq[\lambda]^{\kappa}$ and suppose $P$ is a notion of forcing. We say that $P$ is simply $\mathcal{F}$-proper on the level of $\kappa$ if there is $\theta$ such that whenever

a) $p \in P$,

b) $M \prec H(\theta)$,

c) $p, P, \mathcal{F} \in M$

d) $M \cap \lambda \in \mathcal{F}$,

then there is $p_{0} \leq p$ such that if $q \geq p_{0}$, then there are $\pi_{1}, \ldots, \pi_{k} \in M$ and $a$ formula $\phi\left(x_{0}, x_{1}, \ldots, x_{k}\right)$ which well reflects $q$ in $\left(M, \pi_{1}, \ldots, \pi_{k}\right)$.

Lemma 26. If $P$ is simply $\mathcal{F}$-proper on the level of $\kappa$, then $P$ is $\mathcal{F}$-proper on the level of $\kappa$.

Proof. We will prove that whenever $M, p$ are as in $(a)-(d)$ of Definition 25 then $p_{0}$ is a $(P, M)$-generic condition. Let $D \in M$ be dense, we will show that $D \cap M$ is predense below $p_{0}$. Let $q \leq p_{0}$, we may w.l.o.g. assume that $q \in D$. Let $\pi_{1}, \ldots, \pi_{k} \in M$ and $\phi\left(x_{0}, x_{1}, \ldots, x_{k}\right)$ be such that $\phi\left(x_{0}, x_{1}, \ldots, x_{k}\right)$ well reflects $q$ in $\left(M, \pi_{1}, \ldots, \pi_{k}\right)$. By i) of Definition 24 , we have $\phi\left(q, \pi_{1}, \ldots \pi_{k}\right)$ in $H(\theta)$. By the elementarity $M$ satisfies the formula " $\exists x \in P \phi\left(x, \pi_{1}, \ldots \pi_{k}\right) \& x \in D$ ". So let $s \in M$ witness this fact. Now by Definition $24 s$ and $q$ are compatible, so $D \cap M$ contains a condition compatible with $q$ which proves that $D \cap M$ is predense below $q$ which completes the proof.

3.3. The forcing. Fix a regular $\kappa$, a cardinal $\lambda \geq \kappa^{+}$and a $\left(\kappa^{+}, \lambda\right)$-semimorass $\mathcal{F}$ which is a stationary coding set and assume that $\kappa^{<\kappa}=\kappa$ and $2^{\kappa}=\kappa^{+}$. The consistency of these conditions is proved in [Ksz1].

We consider the following forcing $P$ whose conditions $p$ are of the form $p=\left(a_{p}, f_{p}, A_{p}\right)$ where:

a) $a_{p} \in[\lambda]<\kappa$

b) $f_{p}:\left[a_{p}\right]^{2} \rightarrow \kappa^{+}$.

c) $A_{p} \in[\mathcal{F}]<\kappa$

d) $f_{p}(\alpha, \beta) \leq \operatorname{rank}(X)$ for $X \in A_{p}$ and $\alpha, \beta \in X$.

The order is just the inverse inclusion, i.e., $p \leq q$ if and only if $a_{p} \supseteq a_{q}, f_{p} \supseteq f_{q}$, $A_{p} \supseteq A_{q}$.

Fact 27. $P$ is simply $\mathcal{F}$-proper on the level of $\kappa$.

Proof. Let $\theta=\lambda^{+}$and let $M, p$ be as in $(a)-(d)$ of Definition 25. with $[M]^{<\kappa} \subseteq$ $M$. The existence of such an $M$ follows from our assumptions about $\mathcal{F}$ and the cardinal arithmetic assumptions. Let $X_{0}=M \cap \lambda$. Let $p_{0}=\left(a_{p}, f_{p}, A_{p} \cup\left\{X_{0}\right\}\right)$. 
Finally, let $q \leq p_{0}$. The proof consists of finding the parameters $\pi_{1}, \ldots, \pi_{k} \in M$ and a formula $\phi\left(x_{0}, x_{1}, \ldots, x_{k}\right)$ which well reflects $q$ in $\left(M, \pi_{1}, \ldots, \pi_{k}\right)$.

Define $q \mid M=\left(a_{q} \cap M, f_{q} \mid M, A_{q} \cap M\right)$.

Introduce notation $\delta=M \cap \kappa^{+}=\operatorname{rank}(M)$ (the second equality follows from Fact 19).

Note that $A_{q} \cap M=A_{q \mid M}=\left\{X \in A_{q}: X \in M, X \subset X_{0}\right\}$. This follows from the fact that $\mathcal{F}$ is a stationary coding set i.e., Fact 18 . The assumption that $[M]^{<\kappa} \subseteq M$ implies that $a_{q \mid M}, A_{q \mid M} \in M$. Also as $(d)$ of the definition of the forcing is satisfied for $q \in P$ and $X=X_{0} ; \alpha, \beta \in X_{0}=M \cap \lambda$, we may conclude that $f_{q \mid M}, \in M$, in other words we have $q \mid M \in M \cap P$.

It is clear that $q \mid M \leq p$.

By Fact 20 and the fact that $[M]^{<\kappa} \subseteq M$, in $M$ there is a family $\mathcal{Z}$ of elements of $\mathcal{F}$ such that $\bigcup\left\{X \cap M: \operatorname{rank}(X)<\delta, X \in A_{q}\right\} \subseteq \bigcup \mathcal{Z}$. By the cofinality (which follows from the stationarity) of $\mathcal{F}$ in $[\lambda]^{\kappa}$ and the elementarity of $M$ we may find $Z \in \mathcal{F} \cap M$ such that $\bigcup\left\{X \cap M: \operatorname{rank}(X)<\delta, X \in A_{q}\right\} \subseteq Z$.

Let $\phi\left(x_{0}, x_{1}, x_{2}, x_{3}, x_{4}\right)$ be the formula which says that $x_{0}$ is a condition of the partial order $x_{4}$ which extends in $x_{4}$ the condition $x_{3}$ and such that the difference between the first coordinate of $x_{0}$ and $x_{2}$ is disjoint from $x_{1}$

Subfact 28. $\phi\left(x_{0}, x_{1}, x_{2}, x_{3}, x_{4}\right)$ well-reflects $q$ in $\left(M, Z, a_{q \mid M}, q \mid M, P\right)$. Moreover whenever $\phi\left(s, Z, a_{q \mid M}, q \mid M, P\right)$ holds in $M$, then the common extension $r \leq q, s$ can be chosen to satisfy $f_{r} \mid\left(a_{s}-a_{q \mid M}\right) \otimes\left(a_{q}-a_{q \mid M}\right)=h$ for any function $h:\left(a_{s}-a_{q \mid M}\right) \otimes\left(a_{q}-a_{q \mid M}\right) \rightarrow \delta$.

Proof. It is clear that $\phi\left(q, Z, a_{q \mid M}, q \mid M, P\right)$ holds in $H\left(\lambda^{+}\right)$.

Now let $s \in M$ be a condition satisfying $\phi\left(s, Z, a_{q \mid M}, q \mid M, P\right)$ i.e., $s$ extends in $P$ the condition $q \mid M$ and $a_{s}-a_{q \mid M}$ is disjoint from $Z$, and let $h$ be any function such that

$$
h:\left(a_{s}-a_{q \mid M}\right) \otimes\left(a_{q}-a_{q \mid M}\right) \rightarrow \delta .
$$

Define the common extension $r$ as follows: $a_{r}=a_{s} \cup a_{q}, f_{r}=f_{s} \cup f_{q} \cup h$, $A_{r}=A_{s} \cup A_{q}$. Clearly all clauses of the definition of the forcing $P$ but d) are trivially satisfied for $r$. So let us prove $(d)$. Let $\alpha, \beta \in a_{r}$ and $X \in A_{r}$, we will consider a few cases.

Case 1. $\alpha, \beta \in a_{s}, X \in A_{s}$ It is trivial because $s \in P$.

Case 2. $\alpha, \beta \in a_{q}, X \in A_{q}$ It is trivial because $q \in P$.

Case 3. $\alpha, \beta \in a_{s}, X \in A_{q}$.

Since $\phi\left(s, Z, a_{q \mid M}, q \mid M, P\right)$ holds in $M$, we have that either $\operatorname{rank}(X) \geq \delta=$ $M \cap \kappa^{+}$in which case $(d)$ is satisfied because $f_{r}(\alpha, \beta)=f_{s}(\alpha, \beta)<\delta$ as $s \in M$, or $\operatorname{rank}(X)<\delta$ and then by the definition of $\phi$ and $Z$ we get that $\alpha, \beta \in a_{s} \cap a_{q}$, so we are again in case 2 .

Case 4. $\alpha, \beta \in a_{q}, X \in A_{s}$. 
This means that $\alpha, \beta \in M$, i.e., $\alpha, \beta \in a_{s} \cap a_{q}$ so we are again in case 1 .

Case 5. $\alpha \in a_{s}-a_{q}$ and $\beta \in a_{q}-a_{s}$.

Then $X \in A_{q}$ and so by the definition of $\phi$ and $Z$, we have that $\operatorname{rank}(X) \geq \delta$, but $f_{r}(\alpha, \beta)=h(\alpha, \beta)<\delta$, so $(d)$ is satisfied.

The proof of the subfact completes the proof of Fact 27.

Definition 29. For $p \in P$ by support of $p$, denoted $\operatorname{supp}(p)$ we mean the set $a_{p} \cup \bigcup A_{p}$.

Definition 30. We say that two conditions $p, q$ of $P$ are isomorphic (via $\pi$ : $\operatorname{supp}(p) \rightarrow \operatorname{supp}(q))$ if and only if $\pi: \operatorname{supp}(p) \rightarrow \operatorname{supp}(q))$ is an order preserving bijection constant on $\operatorname{supp}(p) \cap \operatorname{supp}(q)$ and

- $\pi^{\prime \prime}\left[a_{p}\right]=a_{q}$

- $\left\{\pi^{\prime \prime}[X]: X \in A_{p}\right\}=A_{q}$,

- $f_{q}(\pi(\alpha), \pi(\beta))=f_{p}(\alpha, \beta)$ for all $\alpha, \beta \in a_{p}$.

Remark. Note that if $p, q, \pi$ are as in the above definition and $X \in A_{p}$, then $\operatorname{rank}\left(\pi^{\prime \prime}[X]\right)=\operatorname{rank}(X)$.

Lemma 31. Suppose $p, q \in P$ are isomorphic via $\pi: \operatorname{supp}(p) \rightarrow \operatorname{supp}(q)$. Let $\Delta=\operatorname{supp}(p) \cap \operatorname{supp}(q)$ and $r \leq p$ be such that $\operatorname{supp}(r) \cap \operatorname{supp}(q)=\Delta$. Then for any function $h:\left(a_{r}-\Delta\right) \otimes\left(a_{q}-\Delta\right) \rightarrow \kappa^{+}$there is a condition $s \leq r, q$ such that $a_{s}=a_{r} \cup a_{q}, A_{s}=A_{r} \cup A_{q}, f_{s}=f_{r} \cup f_{q} \cup h$.

Proof. The only non-automatic condition of the definition of $P$ which needs to be checked is $(d)$.

Case 1. $\alpha, \beta \in a_{r}$.

If $X \in A_{r}$, we are trivially done. If $X \in A_{q}$ and $\alpha, \beta \in X$, then $\alpha, \beta \in \Delta$, hence $\alpha, \beta \in \pi^{-1}[X] \in A_{p} \subseteq A_{r}$.

Case 2. $\alpha, \beta \in a_{q}$.

Similar to the previous case.

Case 3. $\alpha \in a_{r}-a_{q}, \beta \in a_{q}-a_{r}$.

Note that there cannot be any $X \in A_{s}$ which contains both $\alpha$ and $\beta$, hence $f_{s}(\alpha, \beta)$ can be arbitrary.

Fact 32. Assuming $2^{\kappa}=\kappa^{+}, P$ is $\kappa$-closed and $\kappa^{++}$-c.c. Thus by Fact 27 , Lemma 26 and Fact 23, $P$ preserves cardinals.

Proof. It is clear that $P$ is $\kappa$-closed (the conditions are of sizes less than $\kappa$ and $\kappa$ is regular).

The proof of the $\kappa^{++}$-chain condition is also a standard application of the $\Delta$-system lemma to the sequence of supports $\left\{\operatorname{supp}\left(p_{\xi}\right): \xi<\kappa^{++}\right\}$of some conditions $p_{\xi} \in P$ under our cardinal arithmetic assumption. The natural amalgamation $\left(a_{p_{\xi_{1}}} \cup a_{p_{\xi_{2}}}, f_{p_{\xi_{1}}} \cup f_{p_{\xi_{2}}} \cup h, A_{p_{\xi_{1}}} \cup A_{p_{\xi_{2}}}\right)$ for arbitrary $h$ : $\left(a_{p_{\xi_{1}}}-a_{p_{\xi_{2}}}\right) \otimes\left(a_{p_{\xi_{2}}}-a_{p_{\xi_{2}}}\right) \rightarrow \kappa^{+}$of two isomorphic conditions $p_{\xi_{1}}$ and $p_{\xi_{2}}$ is a condition by the previous lemma. 
Theorem 33. In $V^{P}$ there is a $\lambda \times \lambda$, $\kappa^{+}$-strongly universal matrix and hence a $\kappa^{+}$-strongly unbounded function on $\lambda$.

Proof. Clearly, we claim that $f=\bigcup\left\{f_{p}: p \in G\right\}$ defines such a matrix, where $G$ is a $P$-generic over $V$.

Let $\nu$ be an ordinal less than $\kappa$ and let $B=\left(b_{i, j}\right)_{i, j<\nu}$ be a $\nu \times \nu$ matrix with entries from $\kappa^{+}$. Fix a set $A=\{\dot{a}: a \in A\} \subseteq[\lambda]^{\nu}$ of $P$-names for disjoint $\nu$-tuples $\dot{a}=\left\{\dot{a}_{i}: i<\nu\right\}$ of elements of $\lambda$. Fix a condition $p \in P$.

Take a model $M \prec H\left(\lambda^{+}\right)$such that $M \cap \lambda=X \in \mathcal{F}$ and $p \in P \cap M ; B, \mathcal{F} \in M$ and $\left\{\dot{a}^{\xi}: \xi<\kappa^{+}\right\} \in M$. We will show that there are $\xi_{1}<\xi_{2}<\kappa^{+}$and $r \leq p$ such that

$$
r \Vdash \forall i, j<\nu \quad \dot{f}\left(\dot{a}_{i}^{\xi_{1}}, \dot{a}_{j}^{\xi_{2}}\right)=\check{b}_{i, j}
$$

First take a condition $p_{0} \leq p$ as in Fact 27, i.e., $a_{p}=a_{p_{0}}, f_{p}=f_{p_{0}}, A_{p}=$ $A_{p_{0}} \cup X_{0}$. Take $q \leq p_{0}$ and $\xi_{1} \in \kappa^{++}$such that there is $a \in[\lambda-M]^{<\kappa}$ such that $q \|-\dot{a}^{\xi_{1}}=\check{a}$ and $a \subseteq a_{q}$. This can be done as $\left\{\dot{a}^{\xi}: \xi<\kappa^{+}\right\}$is a sequence of names for disjoint sets and $|M|=\kappa$. proceed as in the proof of Fact 27. Let $s \geq q \mid M$ satisfy $\phi\left(s, Z, a_{q \mid M}, q \mid M, P\right)$ from Subfact 27 . Again by the disjointness of the sets and the elementarity of $M$, we can find an $s$ and $\xi_{2}$ such that $\phi\left(s, Z, a_{q \mid M}, q \mid M, P\right)$ holds in $M$ and moreover there is $b \in[M-Z]^{<\kappa}$ such that $q \| \dot{a}^{\xi_{2}}=\breve{b}$ and $b \subseteq a_{s}$. Now by Fact 28 (the second part) we can design $h$ so that $h \mid(a \otimes b)=B$. Now $r$ as in Fact 28 is an extension of $p$ which forces the required equality.

Finally, note that as $P$ is $\kappa$-closed by Fact 32 , the matrix $B$ can be assumed to be in the ground model.

Remark. By Subfact $28, h$ can be constantly equal to 0 , thus the function we obtained in the case $\kappa=\omega$ does not have the property from Fact 10. Similar arguments show that the natural forcing which adds uncountable family of disjoint finite sets witnessing the failure of the strong unbounded property is c.c.c. thus the function is not c.c.c. indestructible (see Definition 9).

\section{References}

[B] J. Baumgartner, Applications of proper forcing, Handbook of Set-theoretic Topology, edited by K. Kunen and J.E. Vaughan, North-Holland Publishing Co., Amsterdam, (1984), 913-959.

[BS] J. Baumgartner, S. Shelah, Remarks on superatomic Boolean algebras, Ann. Pure. Appl. Logic 33 (1987), 109-129.

[BSp] J. Baumgartner, O. Spinas, Independence and consistency proofs in quadratic form theory, J. Symbolic Logic 56 (1991), 1195-1211.

[Be] M. Bekkali, Topics in set theory, Lebesgue measurability, large cardinals, forcing axioms, rho-functions. Notes on lectures by Stevo Todorčević. Lecture Notes in Mathematics, 1476. Springer-Verlag, Berlin, 1991.

[EGKTT] K. Eda, G. Gruenhage, P.Koszmider, K. Tamano, S. Todorčević, Sequential fans in topology, Topology Appl. 67 (1995), 189-220.

[D] K. Devlin, Aspects of constructibility, Lecture Notes in Mathematics, Vol. 354. Springer-Verlag, Berlin-New York, 1973. 
[K] K. Kunen, Set theory. An introduction to independence proofs, Studies in Logic and the Foundations of Mathematics, 102. North-Holland Publishing Co., AmsterdamNew York, 1980.

[Ka] A. Kanamori, The higher infinite. Large cardinals in set theory from their beginnings, Perspectives in Mathematical Logic. Springer-Verlag, Berlin, 1994.

[Kj] P. Komjath, A set mapping with no infinite free subsets, J. Symbolic Logic 56 (1991), 1400-1402.

[Ksz1] P. Koszmider, Semimorasses and nonreflection at singular cardinals, Ann. Pure Appl. Logic 72 (1995), 1-23.

[Ksz2] _ _ On the existence of strong chains in $\wp\left(\omega_{1}\right) / F i n$, J. Symbolic Logic 63 (1998), 1055-1062.

[Ksz3] , On strong chains of uncountable functions, Isreal J. Math 118 (2000), 289-315.

[Ksz4] _ Banach spaces of large densities but few operators, preprint.

[M] C. Morgan, Morasses, square and forcing axioms, Ann. Pure Appl. Logic 80 (1996), 139-163.

[R] F. Rowbottom, Some strong axioms of infinity incompatible with the axiom of constructibility, Ann. Math. Logic 3 (1971), 1-44.

[S1] S. Shelah, Proper forcing, Lecture Notes in Mathematics, 940. Springer-Verlag, Berlin-New York, 1982.

[S2] S. Shelah, A graph which embeds all small graphs on any large set of vertices, Ann. Pure Appl. Logic 38 (1988), 171-183.

[SS1] S. Shelah, J. Steprans, A Banach space on which there are few operators, Proc. Amer. Math. Soc. 104 (1988), 101-105.

[SS2] _ Extraspecial p-groups, Ann. Pure Appl. Logic 34 (1987), 87-97.

[Si] W. Sierpiński, Sur un problème de la tèorie des relations, Ann. Scuola Norm. Sup. Pisa (2) 2 (1933), 285-287.

[T1] S. Todorčević, Directed sets and cofinal types, Trans. Amer. Math. Soc. 290 (1985), 711-723.

[T2] Partitioning pairs of countable ordinals, Acta Math. 159 (1987), 261-294.

[T3] , Remarks on Martin's axiom and the continuum hypothesis, Canad. J. Math. 43 (1991), 832-851.

[T4] Some partitions of three-dimensional combinatorial cubes, J. Combin. Theory Ser. A 68 (1994), 410-437.

[T5] Coherent sequences, to appear in Handbook of Set Theory.

[Ve] B. Velickovic, Forcing axioms and stationary sets, Adv. Math. 94 (1992), 256-284.

[V1] D. Velleman, Morasses, diamond, and forcing, Ann. Math. Logic 23 (1982), 199281.

[V2] _ Simplified morasses, J. Symbolic Logic 49 (1984), 257-271.

[V3] _ Partitionings pairs of countable sets of ordinals, J. Symbolic Logic 55 (1990), 1019-1021.

[Z] J. Zapletal, Strongly almost disjoint functions, Israel J. Math. 97 (1997), 101-111.

$[\mathrm{Zw}] \quad$ W. Zwicker, $\wp_{\kappa} \lambda$ combinatorics. I. Stationary coding sets rationalize the club filter, Axiomatic set theory (Boulder, CO, 1983), 243-259, Contemp. Math., 31, Amer. Math. Soc., Providence, RI, 1984.

Departmento de Matemática, Universidade de São Paulo, Caixa Postal: 66281, SÃo Paulo, SP, CEP: 05315-970, Brasil.

E-mail address: piotr@ime.usp.br 